\title{
Myostatin: The Missing Link between Sarcopenia and Cardiovascular Disease in Chronic Kidney Disease?
}

\author{
Tomoaki Morioka
}

Department of Metabolism, Endocrinology, and Molecular Medicine, Osaka City University Graduate School of Medicine, Osaka, Japan

\section{See article vol. 27: 1039-1052}

Chronic kidney disease (CKD) is characterized by a high prevalence of cardiovascular disease and, more importantly, a high mortality from cardiovascular disease ${ }^{1,2)}$. Recently, it has been widely accepted that individuals with CKD often present with muscle wasting, malnutrition, inflammation, and their interrelationships, the so-called protein-energy wasting $(\mathrm{PEW})^{3)}$. PEW has gained a lot of attention in recent years because it is closely associated with decreased quality of life and increased mortality and hospitalization, as well as increased atherosclerotic cardiovascular disease, in patients with $\mathrm{CKD}^{3)}$. Recent evidence suggests that systemic inflammation is a common basis and a potential therapeutic target of $\mathrm{PEW}^{4,5)}$; however, the mechanisms underlying PEW, especially the pathway linking muscle wasting and vascular disease, remain poorly understood.

Myostatin, a member of the transforming growth factor (TGF)- $\beta$ superfamily, is predominantly produced in the skeletal muscle in response to stress conditions and negatively regulates skeletal muscle growth $^{6}$. It is currently accepted that myostatin activates several intracellular signaling pathways to inhibit muscle differentiation, decrease protein synthesis, and stimulate protein degradation ${ }^{6}$. The upregulation of myostatin in skeletal muscle has been shown as a major pathway responsible for muscle wasting in patients with advanced $\mathrm{CKD}^{7,8)}$. It is suggested that the activation of myostatin depends on the inflammation to cause muscle wasting in CKD because the upregulation of myostatin is directly associated with the interleukin-6/signal transducer and activator of transcription 3 cascade in CKD muscles ${ }^{7,8)}$.

More recently, several lines of evidence indicate that myostatin and its receptors are expressed not only in skeletal muscle but also in the vasculature and play a role in vascular inflammation, fibrosis, and athero- sclerotic damage ${ }^{6,9)}$. In a study utilizing human specimens, the expression of myostatin was undetectable in normal vessels but became increasingly detectable in vascular smooth muscle cells (VSMCs) and in infiltrating macrophages in progressive atherosclerotic lesions ${ }^{9}$. In vitro studies have further shown that myostatin upregulates monocyte chemoattractant protein (MCP)-1, downregulates cell proliferation, and disorganizes cytoskeletal structure in cultured $\mathrm{VSMCs}^{6,9)}$, suggesting an active role of myostatin in the development of atherosclerosis. Considering that myostatin is upregulated in the skeletal muscle and that it plays a pivotal role in muscle wasting in $\mathrm{CKD}^{7,8)}$, it is reasonable to assume that myostatin also plays a role in vascular diseases associated with CKD; however, only limited data are available regarding the effects of myostatin on the vasculature.

In this issue of the Journal of Atherosclerosis and Thrombosis, Esposito et al. ${ }^{10)}$ utilized vascular specimens obtained from 16 patients with CKD undergoing renal transplantation and examined the expression of myostatin and related factors compared with that of control nonuremic donors. The results demonstrated that the expression of myostatin was upregulated, accompanied by the upregulation of atrogin- 1 and MuRF-1, members of ubiquitin E3 ligases, increased expression of MCP-1, a major pro-inflammatory chemokine, and decreased expression of cytoskeleton protein and Klotho, in the arterial wall of patients with CKD. Moreover, myostatin induced Smad 2/3 phosphorylation and upregulated TGF- $\beta$ and collagen-1 expression in cultured VSMCs, suggesting a profibrotic effect of myostatin on VSMCs ${ }^{11}$. Finally, the main alterations induced by myostatin in the arterial wall of patients with CKD were confirmed in primary human VSMCs exposed to uremic serum. These findings suggest that uremia increases myostatin gene expression and promotes vascular inflammation, ubiquitin-dependent proteolysis, and stiffness in the arterial wall of patients with CKD.

Address for correspondence: Tomoaki Morioka, Department of Metabolism, Endocrinology, and Molecular Medicine, Osaka City University Graduate School of Medicine, 1-4-3, Asahi-machi, Abeno-ku, Osaka 545-8585, JapanＥ-mail: m-tomo@med.osaka-cu.ac.jp

Received: March 20, 2020 Accepted for publication: March 30, 2020

Copyright@2020 Japan Atherosclerosis Society

This article is distributed under the terms of the latest version of CC BY-NC-SA defined by the Creative Commons Attribution License. 


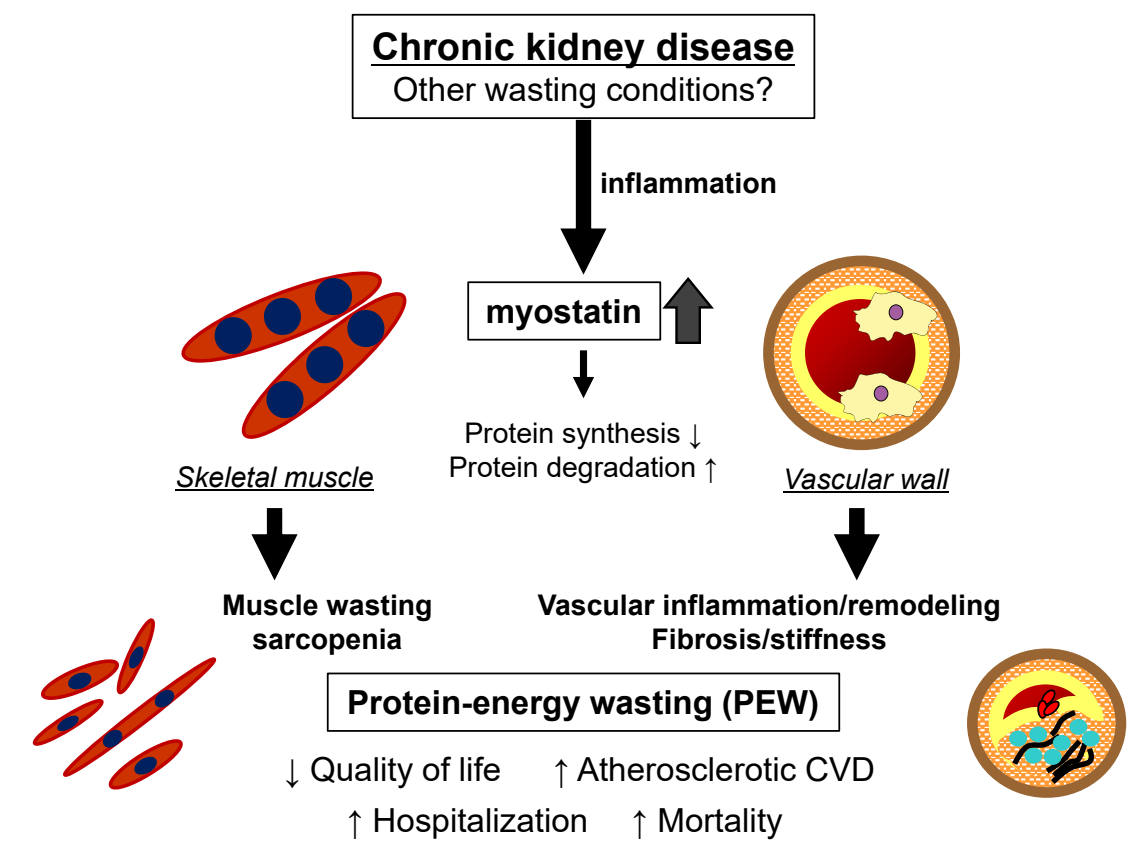

Fig. 1. Schematic representation of the potential role of myostatin activation in the development of sarcopenia and atherosclerosis in patients with chronic kidney disease

CVD, cardiovascular disease.

In the data produced by Esposito et al., despite the upregulation of these genes, it remains unknown why protein expression levels of myostatin and MCP-1 were not increased in the arterial wall of patients with $\mathrm{CKD}^{10)}$. Moreover, another study by Lee et al. just recently reported that lower serum myostatin levels are associated with higher scores of aortic calcification as well as with lower skeletal muscle mass and lower bone mineral density, in 71 patients with CKD undergoing dialysis ${ }^{12}$. Although the authors suggested a protective effect of myostatin against vascular calcification and a close link between decreased skeletal muscle mass and vascular calcification, the exact mechanism responsible for the negative association between serum myostatin levels and vascular calcification remains unclear. Thus far, it remains undetermined how CKD affects the regulation of gene expression, protein expression at the cellular/tissue level, and serum levels of myostatin, all of which need to be revealed in future research.

The study conducted by Esposito et al. ${ }^{10)}$ provided a direct evidence for the involvement of myostatin in CKD-induced inflammation, protein degradation, and vascular remodeling in the arterial wall. Considering that myostatin plays a major role in the development of skeletal muscle wasting in $\mathrm{CKD}^{13)}$, the study by Esposito et al. suggested that the activation of myostatin is a possible link between inflamma- tion, vascular disease, and skeletal muscle wasting in the context of CKD (Fig. 1). Further studies are warranted to confirm this hypothesis in a population of patients with CKD and a wide range of glomerular filtration ratio. Moreover, it is interesting to speculate that the activation of myostatin is a common pathway linking muscle wasting and vascular disease not only in CKD but also in other wasting conditions such as cancer and cardiac cachexia.

\section{Conflict of Interest}

None.

\section{References}

1) Wanner C, Amann $K$ and Shoji T: The heart and vascular system in dialysis. Lancet, 2016; 388: 276-284

2) Kinoshita M, Yokote $K$, Arai $H$, Iida $M$, Ishigaki $Y$, Ishibashi S, Umemoto S, Egusa G, Ohmura H, Okamura T, Kihara S, Koba S, Saito I, Shoji T, Daida H, Tsukamoto K, Deguchi J, Dohi S, Dobashi K, Hamaguchi H, Hara M, Hiro T, Biro S, Fujioka Y, Maruyama C, Miyamoto Y, Murakami Y, Yokode M, Yoshida H, Rakugi H, Wakatsuki A, Yamashita S, Committee for Epidemiology and Clinical Management of Atherosclerosis: Japan Atherosclerosis Society (JAS) Guidelines for Prevention of Atherosclerotic Cardiovascular Diseases 2017. J Atheroscler Thromb, 2018; 25: 846-984 
3) Fouque D, Kalantar-Zadeh K, Kopple J, Cano N, Chauveau P, Cuppari L, Franch H, Guarnieri G, Ikizler TA, Kaysen G, Lindholm B, Massy Z, Mitch W, Pineda E, Stenvinkel P, Trevino-Becerra A and Wanner C: A proposed nomenclature and diagnostic criteria for proteinenergy wasting in acute and chronic kidney disease. Kidney Int, 2008; 73: 391-398

4) Miki Y, Morioka T, Shioi A, Fujimoto K, Sakura T, Uedono H, Kakutani Y, Ochi A, Mori K, Shoji T, Emoto $\mathrm{M}$ and Inaba $\mathrm{M}$ : Oncostatin M induces C2C12 myotube atrophy by modulating muscle differentiation and degradation. Biochem Biophys Res Commun, 2019; 516: 951956

5) Machowska A, Carrero JJ, Lindholm B and Stenvinkel P: Therapeutics targeting persistent inflammation in chronic kidney disease. Transl Res, 2016; 167: 204-213

6) Verzola D, Barisione C, Picciotto D, Garibotto G and Koppe L: Emerging role of myostatin and its inhibition in the setting of chronic kidney disease. Kidney Int, 2019; 95: 506-517

7) Zhang L, Pan J, Dong Y, Tweardy DJ, Dong Y, Garibotto $G$ and Mitch WE: Stat3 activation links a C/EBPdelta to myostatin pathway to stimulate loss of muscle mass. Cell Metab, 2013; 18: 368-379

8) Verzola D, Procopio V, Sofia A, Villaggio B, Tarroni A, Bonanni A, Mannucci I, De Cian F, Gianetta E, Saffioti S and Garibotto G: Apoptosis and myostatin mRNA are upregulated in the skeletal muscle of patients with chronic kidney disease. Kidney Int, 2011; 79: 773-782

9) Verzola D, Milanesi S, Bertolotto M, Garibaldi S, Villaggio B, Brunelli C, Balbi M, Ameri P, Montecucco F, Palombo D, Ghigliotti G, Garibotto G, Lindeman JH and Barisione C: Myostatin mediates abdominal aortic atherosclerosis progression by inducing vascular smooth muscle cell dysfunction and monocyte recruitment. Sci Rep, 2017; 7: 46362

10) Esposito P, Verzola D, Porta E, Milanesi S, Grignano MA, Avella A, Gregorini M, Abelli M, Ticozzelli E, Rampino T and Garibotto G: Myostatin in the Arterial Wall of Patients with End-Stage Renal Disease. J Atheroscler Thromb, 2020; 27: 1039-1052

11) Yamashiro $Y$ and Yanagisawa $H$ : Crossing Bridges between Extra- and Intra-Cellular Events in Thoracic Aortic Aneurysms. J Atheroscler Thromb, 2018; 25: 99-110

12) Lee SM, Kim SE, Lee JY, Jeong HJ, Son YK and An WS: Serum myostatin levels are associated with abdominal aortic calcification in dialysis patients. Kidney Res Clin Pract, 2019; 38: 481-489

13) Wang XH and Mitch WE: Mechanisms of muscle wasting in chronic kidney disease. Nat Rev Nephrol, 2014; 10: 504-516 